\title{
Assessment of left atrial systolic dyssynchrony in paroxysmal atrial fibrillation and heart failure using cardiac magnetic resonance imaging: MESA study
}

\author{
Luisa A Ciuffo ${ }^{1,2^{*}}$, Ravi Sharma ${ }^{1}$, Mohammadali Habibi ${ }^{6}$, Bharath Ambale Venkatesh ${ }^{1}$, Boaz D Rosen ${ }^{1}$, \\ Masamichi Imai ${ }^{1}$, Steven Shea ${ }^{5}$, Robyn McClelland ${ }^{4}$, Colin O Wu ${ }^{3}$, Susan R Heckbert ${ }^{4}$, David Bluemke ${ }^{3}$, \\ Joao A Lima'
}

From 18th Annual SCMR Scientific Sessions

Nice, France. 4-7 February 2015

\section{Background}

Left atrial (LA) remodeling in response to cardiovascular and hemodynamic stress may precede atrial fibrillation (AF) and heart failure (HF). We hypothesized that LA systolic synchronous contraction as a functional measure of LA remodeling is deranged in patients with paroxysmal AF and HF.

\section{Methods}

We performed a nested case-control analysis with 1:2 matching for 39 cases of paroxysmal AF $(n=28$, in sinus rhythm during cardiac magnetic resonance (CMR)) and HF $(n=14, A F+H F ; n=3)$ and 78 controls with similar demographic and clinical characteristics at the baseline (Table 1). LA circumferential (short axis) and longitudinal strain rate (horizontal long axis) were measured using Multi-modality Tissue Tracking (Toshiba, Japan) from short and long-axis cine CMR images. Circumferential LA systolic dyssynchrony among 18 LA segments (6 segments x 3 slices) was evaluated as; Standard Deviation (SD) of time to pre atrial contraction Strain rate $\left(\mathrm{PreA} \mathrm{Sr}^{\mathrm{c}}\right)$ and Peak systolic strain rate $\left(\right.$ Peak $\mathrm{Sra}^{\mathrm{c}}$ ) (Figure 1). Similarly, longitudinal LA dyssynchrony parameters (among 6 segments) were: SD-Time to pre-atrial contraction strain rate $\left(\operatorname{PreA~Sr}{ }^{\mathrm{L}}\right.$ ) and SD-Time to peak systolic strain rate (Peak$\mathrm{Sra}^{\mathrm{L}}$ ). Wilcoxon-rank sum test (non-parametric) or two sample t-test (parametric) were used for comparison between the groups.

\section{Results}

In participants during MESA exam 5 (age $74 \pm 8$ years, $51.4 \%$ men), systolic circumferential dyssynchrony (SD$\mathrm{TP}-\mathrm{PreA} \mathrm{Sr}^{\mathrm{c}}, \mathrm{msec}$ ) was significantly higher in the cases compared to controls (45.06 vs. 28.73, p<0.010). Similarly,

Table 1 Left atrial circumferential and longitudinal systolic dyssynchrony parameters among the cases and the control group.

\begin{tabular}{|c|c|c|c|c|c|c|c|}
\hline \multirow{2}{*}{$\begin{array}{c}\text { Parameters } \\
\text { Longitudinal }\end{array}$} & \multicolumn{3}{|c|}{ Controls $(n=78)$} & \multicolumn{3}{|c|}{ Cases $(n=39)$ (paroxysmal AF + Heart Failure) } & \multirow[t]{2}{*}{ p-value } \\
\hline & Mean \pm SD & Median & IQR & Mean \pm SD & Median & IQR & \\
\hline SD-TP PreA SrL, msec & $36.43 \pm 33.53$ & 27.40 & $15.50-46.13$ & $51.62 \pm 33.40$ & 39.70 & $32.07-61.78$ & 0.001 \\
\hline SD-TP Peak SraL & $35.92 \pm 43.22$ & 23.86 & $16.32-35.60$ & $45.23 \pm 42.04$ & 32.50 & $22.98-44.54$ & 0.027 \\
\hline \multicolumn{8}{|l|}{ Circumferential } \\
\hline SD-TP PreA Src, msec & $28.73 \pm 13.90$ & 26.23 & $19.70-34.98$ & $45.06 \pm 30.25$ & 35.49 & $24.84-57.03$ & 0.010 \\
\hline SD-TP Peak Srac, msec & $28.95 \pm 23.1$ & 23.04 & $16.46-31.57$ & $36.46 \pm 30.93$ & 26.83 & $17.45-44.02$ & 0.316 \\
\hline LA maximum volume/BSA & $33.79 \pm 9.57$ & 31.72 & $28.64-38.86$ & $45.02 \pm 17.85$ & 44.38 & $31.04-56.44$ & $<0.0001$ \\
\hline Sra $(\% / m s)$ & $-1.85 \pm 0.85$ & $\begin{array}{l}-1.74 \\
\end{array}$ & $-2.18:-1.45$ & $-1.03 \pm 0.61$ & -1.04 & $-1.04:-0.53$ & $<0.0001$ \\
\hline
\end{tabular}

Participants were matched for: Age, gender, race/ethnicity.

\footnotetext{
${ }^{1} J o h n s$ Hopkins, Baltimore, MD, USA

Full list of author information is available at the end of the article
} 


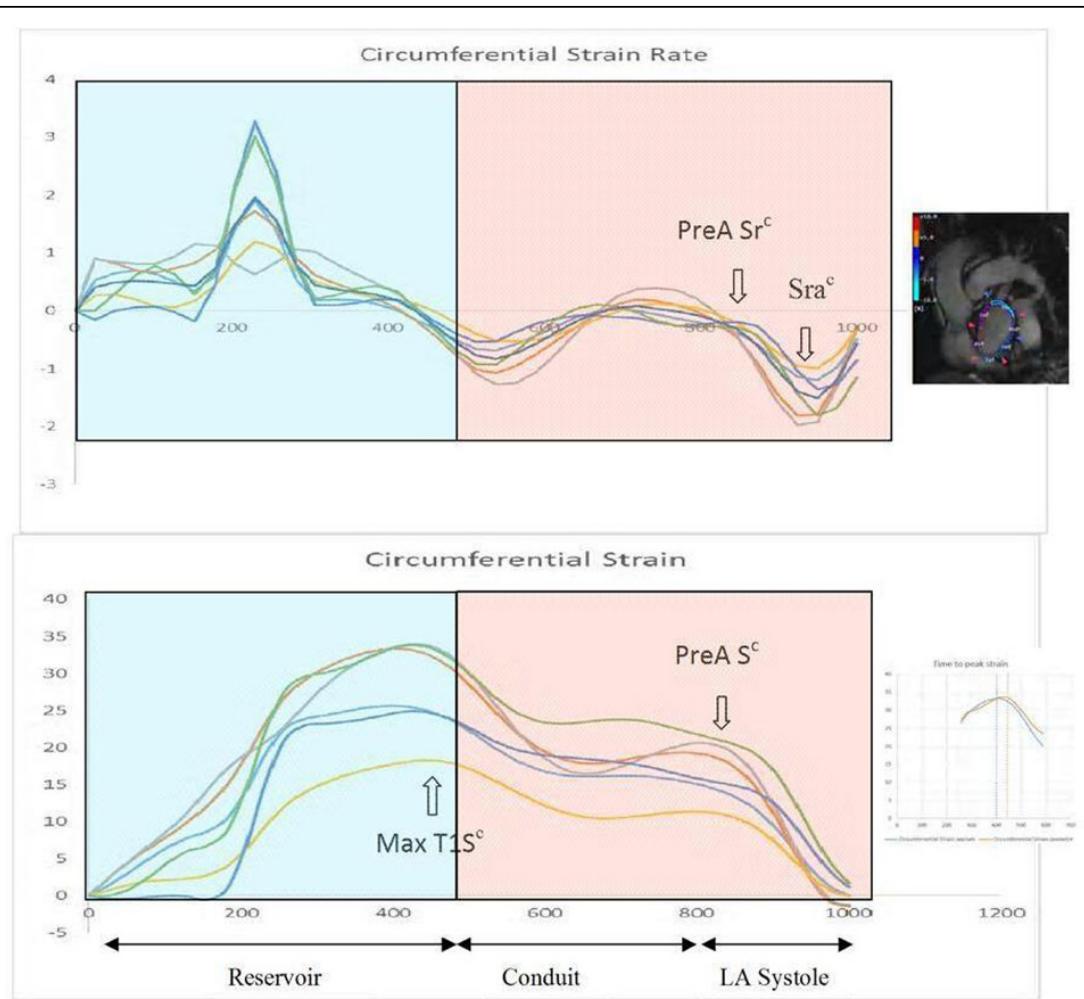

Figure 1 LA circumferential (panel A) and longitudinal (panel B) strain rate curves during a cardiac cycle using multimodality tissue tracking.

case group had greater longitudinal dyssynchrony than controls; SD-TP PreA Sr${ }^{\mathrm{L}}$ (51.62 vs. 36.43, $\left.\mathrm{p}=0.001\right)$ and SD-TP-Peak Sra ${ }^{\mathrm{L}}$ (45.23 vs. 35.92, $\mathrm{p}=0.027$ ) (Table 1).

\section{Conclusions}

Patients with paroxysmal atrial fibrillation and heart failure have significantly higher LA circumferential and longitudinal systolic dyssynchrony compared to normal controls.

\section{Funding}

N/A.

\section{Authors' details}

'Johns Hopkins, Baltimore, MD, USA. ² nniversidade federal da Bahia, Salvador, Brazil. ${ }^{3}$ National Institutes of Health, Bethesda, MD, USA. ${ }^{4}$ University of Washington, Washington, DC, USA. ${ }^{5}$ Columbia University, New York, NY, USA. ${ }^{6}$ Union memorial Hospital, Baltimore, MD, USA.

Published: 3 February 2015

doi:10.1186/1532-429X-17-S1-P322

Cite this article as: Ciuffo et al:: Assessment of left atrial systolic dyssynchrony in paroxysmal atrial fibrillation and heart failure using cardiac magnetic resonance imaging: MESA study. Journal of

Cardiovascular Magnetic Resonance 2015 17(Suppl 1):P322.

\section{Submit your next manuscript to BioMed Central} and take full advantage of:

- Convenient online submission

- Thorough peer review

- No space constraints or color figure charges

- Immediate publication on acceptance

- Inclusion in PubMed, CAS, Scopus and Google Scholar

- Research which is freely available for redistribution 